\title{
Indifference Pricing and Hedging for Volatility Derivatives
}

\author{
M. R. GRASSELLI* \& T. R. HURD* \\ Department of Mathematics and Statistics, McMaster University, Hamilton, Canada
}

(Received 9 March 2006; in revised form 27 July 2006)

\begin{abstract}
Utility based indifference pricing and hedging are now considered to be an economically natural method for valuing contingent claims in incomplete markets. However, acceptance of these concepts by the wide financial community has been hampered by the computational and conceptual difficulty of the approach. This paper focuses on the problem of computing indifference prices for derivative securities in a class of incomplete stochastic volatility models general enough to include important examples. A rigorous development is presented based on identifying the natural martingales in the model, leading to a nonlinear Feynman-Kac representation for the indifference price of contingent claims on volatility. To illustrate the power of this representation, closed form solutions are given for the indifference price of a variance swap in the standard Heston model and in a new "reciprocal Heston" model. These are the first known explicit formulas for the indifference price for a class of derivatives that is important to the finance industry.
\end{abstract}

KEY WoRdS: Volatility risk, exponential utility, Heston model, variance swap, incomplete markets, certainty equivalent, volatility derivative

\section{Introduction}

It has been amply discussed in recent literature by such authors as Cvitanić, Schachermayer and Wang (2001), Delbaen et al. (2002), Henderson (2002), Musiela and Zariphopoulou (2004), that utility methods provide an economically justified framework for pricing derivatives in incomplete markets, where the traditional paradigms of risk neutral valuation and replication are replaced by the concepts of indifference pricing and hedging introduced by Hodges and Neuberger (1989). The purpose of the present contribution to this field is twofold: first, to provide a class of realistic and financially relevant examples where indifference pricing and hedging can be rigorously and fully solved and these concepts explored in detail; and second, to provide a new way to understand volatility derivatives, which have recently

\footnotetext{
* Research supported by the Natural Sciences and Engineering Research Council of Canada and MITACS, Mathematics of Information Technology and Complex Systems Canada

Correspondence Address: M. R. Grasselli, Department of Mathematics and Statistics, McMaster University, Hamilton, ON, L8S 4K1, Canada. Email: grasselli@math.mcmaster.ca
} 
become an important class of financial products (see Friz and Gatheral, 2005; Carr et al., 2005). Along the way, to keep the narrative interesting, we use a new and direct duality argument to derive the basic indifference results for two dimensional models, instead of using by now well-understood arguments involving PDEs.

We consider stochastic volatility models of the form

$$
\begin{aligned}
& \mathrm{d} S_{t}=S_{t}\left[(\mu-r) \mathrm{d} t+\sqrt{Y_{t}} \mathrm{~d} W_{t}\right], \\
& \mathrm{d} Y_{t}=a\left(t, Y_{t}\right) \mathrm{d} t+b\left(t, Y_{t}\right)\left[\rho \mathrm{d} W_{t}+\bar{\rho} \mathrm{d} Z_{t}\right],
\end{aligned}
$$

for initial values $S_{0}, Y_{0}>0, \mu-r>0$, independent one-dimensional Brownian motions $W_{t}$ and $Z_{t}$ generating a standard filtration $\left(\mathcal{F}_{t}\right)_{0 \leq t \leq T}$ on the probability space $(\Omega, \mathcal{F}, P)$, and correlation parameters $\rho$ and $\bar{\rho}=\sqrt{1-\rho^{2}}$. Here $S_{t}$ denotes the discounted stock price at time $t$, and $Y_{t}=\sigma_{t}^{2}$ is interpreted as the stochastic squared volatility of $S_{t}$. We now make the following standing assumptions:

Assumption 1.1. The coefficient functions $a, b$ are such that (1) has a unique strong solution $\left(S_{t}, Y_{t}\right)$ which lies in $\mathbb{R}_{+} \times \mathbb{R}_{+}$for all $t \in[0, T], P-$ a.s. The correlation parameters $\rho \in(-1,1), \bar{\rho}=\sqrt{1-\rho^{2}}$, mean rate of return $\mu$, and interest rate $r$ are constant.

Assumption 1.2. The discounted contingent claim $B$ has the form $B_{T}=B\left(Y_{T}, V_{T}\right)$, where

$$
V_{t}=\int_{0}^{t} f\left(Y_{s}\right) \mathrm{d} s
$$

and $B: \mathbb{R}_{+} \times \mathbb{R}_{+} \times \mathbb{R}_{+} \rightarrow \mathbb{R}, f: \mathbb{R}_{+} \rightarrow \mathbb{R}_{+}$are deterministic functions such that

$$
E\left[e^{(\gamma+\varepsilon) B}\right]<\infty \quad \text { and } \quad E\left[e^{-\varepsilon B}\right]<\infty \quad \text { for some } \varepsilon>0 .
$$

Assumption 1.3. The market price of risk process, defined by

$$
\lambda\left(t, Y_{t}\right):=\frac{\mu-r}{\sqrt{Y_{t}}}
$$

is such that the density

$$
\frac{\mathrm{d} \tilde{Q}}{\mathrm{~d} P}=\exp \left(-\frac{1}{2} \int_{0}^{T} \lambda_{s}^{2} \mathrm{~d} s-\int_{0}^{T} \lambda_{s} \mathrm{~d} W_{s}\right)
$$

gives rise to a well-defined equivalent local martingale measure $\tilde{Q}$ with finite relative entropy with respect to $P$.

Observe that Assumption 1.1 is weaker than the usual global Lipschitz continuity and growth conditions that are sufficient for the existence of a strong solution $\left(S_{t}\right.$, $\left.Y_{t}\right)$. This avoids ruling out from the start some of the most popular stochastic volatility models, such as the Heston model, for which $Y_{t}$ is a square-root diffusion that does not satisfy the global Lipschitz condition. Moreover, our volatility function 


$$
\sigma\left(t, Y_{t}\right)=\sqrt{Y_{t}}
$$

is neither bounded from above nor bounded away from zero, as is assumed for instance in Sircar and Zariphopoulou (2005). Thus, we allow for the introduction of realistic stochastic volatility models at the expense of having to verify the existence of unique strong solutions to (1) case by case.

Regarding the claim $B$, observe that Assumption 1.2 is significantly weaker than requiring $B$ to be bounded. While boundedness of $B$ is useful in order to assert regularity and growth properties for the indifference prices, it automatically excludes most of the volatility derivatives that are actually traded in financial markets. Two important examples of such derivatives are the variance swap, obtained when

$$
B_{T}=e^{-r T} \int_{0}^{T} Y_{t} \mathrm{~d} t
$$

and the volatility swap, obtained when

$$
B_{T}=e^{-r T} \sqrt{\int_{0}^{T} Y_{t} \mathrm{~d} t} .
$$

These derivatives would be ruled out from the beginning if we assumed that the claim $B$ is bounded; instead, we require the weaker integrability condition (2), which is sufficient to develop the indifference pricing framework. Given a particular market model, we must then verify that the claims of interest satisfy (2), for example by exploring the distributional properties of the process $Y_{t}$.

Finally, Assumption 1.3 is necessary to our analysis since, as we are going to see, the indifference price for volatility derivatives can be expressed in terms of $\tilde{Q}$-expectations. The measure $\tilde{Q}$ is known as the minimal martingale measure and has been investigated in the context of indifference pricing in several of the references quoted above. Although Assumption 1.3 is slightly stronger than requiring viability of the market (in the sense of existence of an equivalent martingale measure), it can also be directly verified for the stochastic volatility models of interest to us.

The remainder of the paper is organized as follows. In Section 2, we survey known results for the optimal hedging problem and show how it leads to the concepts of certainty equivalent and indifference price of a claim as introduced by Hodges and Neuberger (1989). We list sufficient conditions for the existence of an optimal hedge specific to the case of an exponential utility $U(x)=-e^{-\gamma x}$ with risk aversion parameter $\gamma>0$. Proposition 3.1 in Section 3 then states how the utility determines the volatility risk premium associated with each contingent claim $B\left(Y_{T}, V_{T}\right)$, as well as the form of the optimal hedge portfolio in terms of the certainty equivalent process. In Section 4 we prove Theorem 4.1 and its corollary, our main results, which give a generalized nonlinear Feynman-Kac representation for the indifference price of volatility derivatives. This generalizes a result obtained in Tehranchi (2004) for the case of a bounded volatility $\sigma_{t}$ and a claim satisfying a stronger condition than 1.2 (namely having finite moments of all orders). In Section 5, we apply this representation to the variance swap under two different models, the Heston model and "reciprocal Heston" model, and derive explicit formulas for the indifference 
price in terms of the confluent hypergeometric function. Finally, in Section 6 we provide a numerical illustration of some simple ways the indifference price of a variance swap differs from alternative pricing methods.

\section{Utility Based Pricing with Exponential Preferences}

\section{Optimal Hedging Problem}

The central definition in this subject is that of an optimal hedging portfolio for an investor trading in the market defined by the discounted asset process (1). It corresponds to the self-financing strategy followed by investors withinitial wealth $x$ who, when faced with a (discounted) financial liability $B$ maturing at a future time $T$, try to maximize their expected utility of terminal wealth by solving the stochastic control problem

$$
u(x)=\inf _{H \in \mathcal{A}} E\left[e^{-\gamma\left(X_{T}-B\right)} \mid X_{0}=x\right],
$$

where $\gamma>0$ is the risk-aversion parameter. Here $X_{T}$ is the discounted terminal wealth obtained when at each time $t$ one holds $H_{t}$ units in the traded asset and $e^{r t}\left(X_{t}-H_{t} S_{t}\right)$ dollars in a riskless money market account. The discounted wealth process $X_{t}$ satisfies the self-financing condition

$$
\mathrm{d} X_{t}=H_{t} \mathrm{~d} S_{t}=H_{t} S_{t}\left[(\mu-r) \mathrm{d} t+\sigma\left(t, Y_{t}\right) \mathrm{d} W_{t}\right], \quad t \in(0, T),
$$

for a control process $H \in \mathcal{A}$. To complete the specification of the admissible portfolios $\mathcal{A}$, let $\mathcal{M}^{a}(S)$ and $\mathcal{M}^{e}(S)$ denote respectively the sets of absolutely continuous and equivalent local martingale measures for $S$ and let $\mathcal{M}^{f}(S)$ denote the set of measures $Q \in \mathcal{M}^{a}(S)$ with finite relative entropy with respect to $P$. Denoting by $L(S)$ the set of predictable $S$-integrable processes, we follow Becherer (2004) and take the set of admissible portfolios to be

$$
\mathcal{A}=\left\{H \in L(S): \int_{0}^{t} H_{u} \mathrm{~d} S_{u} \text { is a } \mathrm{Q}-\text { martingale for all } \mathrm{Q} \in \mathcal{M}^{f}\right\} .
$$

As noted in the introduction, Assumption 1.3 implies that the set $\mathcal{M}^{e} \cap \mathcal{M}^{f}$ is nonempty, so that the market is viable.

Now, under the condition for the claim $B$ specified in Assumption 1.2, it follows from convex duality (see Becherer, 2001, 2004; Delbaen et al., 2002; Kabanov and Stricker, 2002; Owen, 2002) that the optimal hedging problem (7) has a unique solution $H^{B} \in \mathcal{A}$ satisfying

$$
\gamma e^{-\gamma\left(x+\int_{0}^{T} H^{B} \mathrm{~d} S-B\right)}=\xi \frac{\mathrm{d} Q^{B}}{\mathrm{~d} P},
$$

where $\xi=u^{\prime}(x)$ and $Q^{B} \in \mathcal{M}^{f} \cap \mathcal{M}^{e}$ is the unique maximizer of the corresponding dual problem

$$
\sup _{Q \in \mathcal{M}^{f}} E^{Q}\left[\gamma B-\log \left(\frac{\mathrm{d} Q}{\mathrm{~d} P}\right)\right] .
$$




\section{The Indifference Price and the Certainty Equivalent}

An agent with exponential utility $U(x)=-e^{-\gamma x}$ and initial wealth $x$ will charge a premium for issuing a liability $B$ maturing at $T$. The seller's indifference price for the claim $B$ is defined to be the premium that makes the agent indifferent in the expected utility sense between making the deal or not, that is, the unique solution $\pi^{B}$ to the equation

$$
\min _{H \in \mathcal{A}} E\left[e^{-\gamma\left(x+\int_{0}^{T} H \mathrm{~d} S\right)}\right]=\min _{H \in \mathcal{A}} E\left[e^{-\gamma\left(x+\pi^{B}+\int_{0}^{T} H \mathrm{~d} S-B\right)}\right] .
$$

It is convenient to express the indifference price in terms of the certainty equivalent process $\left(c_{t}^{B}\right)_{0 \leq t \leq T}$ for the claim $B$. Denoting by $H^{B}$ the unique solution to (7), then for each $t \leqslant T$, we can define $c_{t}^{B}$ as the unique solution of

$$
e^{-\gamma\left(X_{t}-c_{t}^{B}\right)}=E\left[e^{-\gamma\left(X_{t}+\int_{t}^{T} H^{B} \mathrm{~d} S-B\right)} \mid \mathcal{F}_{t}\right],
$$

where $X_{t}$ is the wealth obtained from an initial value $x$ by trading according to any admissible portfolio $H$ up to time $t$. In the case $B=0$, then the optimal hedging problem becomes the Merton optimal investment problem and we denote the certaintyequivalent process by $c_{t}^{0}$. From (11), it is clear that $Q^{0}$ is the local martingale measure that minimizes the relative entropy with respect to $P$ and is therefore called the minimal entropy martingale measure for the market.

The conditional version of (12) is thus equivalent to

$$
e^{-\gamma\left(X_{t}-c_{t}^{0}\right)}=e^{-\gamma\left(X_{t}+\pi_{t}^{B}-c_{t}^{B}\right)}
$$

so that the indifference price process is given by

$$
\pi_{t}^{B}=c_{t}^{B}-c_{t}^{0}, \quad t \in[0, T] .
$$

\section{Volatility Risk Premium and the Hedging Portfolio}

As we have seen in the previous section, the solution of the hedging problem (7) through convex duality involves finding a local martingale measure $Q^{B}$ solving the dual problem (11). This optimal measure is not, however, a direct generalization of the risk neutral pricing measure obtained for complete markets. First of all, it depends on the claim $B$ itself, so even for multiples of the same claim we can obtain different optimal measures. Secondly, the indifference price $\pi^{B}$ is not obtained from $Q^{B}$ by a simple expectation (see equation (2.8) in Becherer, 2004). Nevertheless, the optimal measure $Q^{B}$ induces a market price of risk associated with the claim $B$ as follows.

Given the optimal measure $Q^{B}$ associated with the claim $B$, consider the density process 


$$
\Lambda_{t}^{B}:=E\left[\frac{\mathrm{d} Q^{B}}{\mathrm{~d} P} \mid \mathcal{F}_{t}\right],
$$

which, being a strictly positive martingale, can be expressed as the solution of

$$
\frac{\mathrm{d} \Lambda_{t}^{B}}{\Lambda_{t}^{B}}=-\left[\lambda_{t}^{B} \mathrm{~d} W_{t}+v_{t}^{B} \mathrm{~d} Z_{t}\right]
$$

for adapted processes $\lambda_{t}^{B}$ and $v_{t}^{B}$. Since $Q^{B} \in \mathcal{M}^{e} \cap \mathcal{M}^{f}$, the discounted price $S_{t}$ is a local $Q^{B}$-martingale and hence we must have that

$$
\lambda_{t}^{B}=\lambda_{t}:=\frac{\mu-r}{\sqrt{Y_{t}}} .
$$

The remaining process $v_{t}^{B}$ completes the specification of the utility based market price of risk associated with the claim $B$.

We next prove a simple proposition giving the general functional form for the market price of risk and optimal portfolio associated with the claim $B$, valid whenever the certainty equivalent $c^{B}$ is sufficiently regular.

Proposition 3.1. Under Assumptions 1.1, 1.2, 1.3 and the further assumption that the certainty equivalent process is given by $c_{t}^{B}=c^{B}\left(t, Y_{t}, V_{t}\right)$ where the function $c^{B}$ is twice differentiable with respect to $y \in \mathrm{R}_{+}$and continuously differentiable with respect to $(t, v) \in[0, T] \times \mathrm{R}$, we have that

$$
v^{B}(t, y, v)=-\gamma b(t, y) \bar{\rho} \partial_{y} c^{B}(t, y, v),
$$

and the unique optimizer $H_{t}^{B}=h^{B}\left(t, S_{t}, Y_{t}, V_{t}\right)$ for the hedging problem (7) is

$$
h^{B}(t, s, y, v)=\frac{b(t, y) \rho}{s \sqrt{y}} \partial_{y} c^{B}(t, y, v)+\frac{(\mu-r)}{\gamma s y} .
$$

Proof. It follows from (10) that

$$
\begin{aligned}
\Lambda_{t}^{B} & =\frac{1}{\xi} E\left[U^{\prime}\left(X_{T}^{B}-B\right) \mid \mathcal{F}_{t}\right] \\
& =\frac{\gamma e^{-\gamma X_{t}^{B}}}{\xi} E\left[\exp \left(-\gamma \int_{t}^{T} H^{B} \mathrm{~d} S+\gamma B\right) \mid \mathcal{F}_{t}\right] \\
& =\frac{e^{-\gamma\left(X_{t}^{B}-c_{t}^{B}\right)}}{e^{-\gamma\left(x-c_{0}^{B}\right)}},
\end{aligned}
$$

where we have used the definition of the certainty equivalent process $c_{t}^{B}$ and the fact that $\xi=u^{\prime}(x)=U^{\prime}\left(x-c_{0}^{B}\right)$. Applying Itô's lemma to the process $\Lambda_{t}^{B}$ above, we obtain

$$
\frac{\mathrm{d} \Lambda_{t}^{B}}{\Lambda_{t}^{B}}=(\cdots) \mathrm{d} t-\gamma\left[\left(H^{B}-\partial_{s} c^{B}\right) \sqrt{y} s-b \rho \partial_{y} c^{B}\right] \mathrm{d} W+\gamma b \partial_{y} c^{B} \bar{\rho} \mathrm{d} Z
$$

Comparing this with (17) and (18) gives the result. 
Since $\Lambda_{t}^{B}$ is a martingale, the drift term in (22) must vanish. Using this fact and the expression for $H^{B}$ in terms of $c^{B}$ obtained in (20), we are able to derive immediately the partial differential equation satisfied by the certainty equivalent. This is presented in the next corollary and, except for the new linear term associated with the integrated volatility, coincides with previous results obtained from stochastic control via the dynamic programming principle (see for example Sircar and Zariphopoulou, 2005).

Corollary 3.2. Under the conditions of Proposition 3.1, the certainty equivalent process $c_{t}^{B}=c^{B}\left(t, Y_{t}, V_{t}\right)$ satisfies

$$
\partial_{t} c^{B}+\left[a-\frac{b \rho(\mu-r)}{\sqrt{y}}\right] \partial_{y} c^{B}+\frac{1}{2} b^{2} \partial_{y y}^{2} c^{B}+f \partial_{\nu} c^{B}-\frac{(\mu-r)^{2}}{2 \gamma y}+\frac{1}{2} \gamma \bar{\rho} b^{2}\left(\partial_{y} c^{B}\right)^{2}=0
$$

with terminal condition $c^{B}(T, y, v)=B(y, v)$.

As noted in section 2.2, the solution to Merton's problem leads to the minimal entropy martingale measure $Q^{0}$, whose density process $\Lambda_{t}^{0}:=E\left[\frac{\mathrm{d} Q^{0}}{\mathrm{~d} P} \mid \mathcal{F}_{t}\right]$ can be written as

$$
\frac{\mathrm{d} \Lambda_{t}^{0}}{\Lambda_{t}^{0}}=-\left[\lambda_{t} \mathrm{~d} W_{t}+v_{t}^{0} \mathrm{~d} Z_{t}\right]
$$

where $v^{0}$ is obtained from (19) by setting $B=0$, that is

$$
v^{0}(t, y)=-\gamma b \bar{\rho} \partial_{y} c^{0} .
$$

Following Musiela and Zariphopoulou (2004), we define the residual risk associated with the claim $B$ as the difference $\left(X_{t}^{B}-X_{t}^{0}\right)-\pi_{t}^{B}$. Here $X_{t}^{0}$ is the optimal wealth for Merton's problem starting at $x$ and $X_{t}^{B}$ is the optimal wealth for the hedging problem starting at $x+\pi_{0}^{B}$, so that the residual risk at initial time is zero. For the case where the volatility function is taking to be a constant $\sigma$ (so that the stochastic volatility model (1) reduces to a model for a traded asset $S_{t}$ that is correlated to a nontraded asset $\left.Y_{t}\right)$ and a bounded claim of the form $B=B\left(Y_{T}\right)$, these authors were able to show that the process $e^{-\gamma\left(X_{t}^{B}-X_{t}^{0}-\pi_{t}^{B}\right)}$ is a martingale under the physical measure $P$. In the next proposition, we show how this result needs to be modified for the stochastic volatility model (1) and claim $B=B\left(Y_{T}, V_{T}\right)$.

Proposition 3.3. Suppose the conditions of Proposition 3.1 apply to both $c^{B}$ and $c^{0}$. Then the process $e^{-\gamma\left(X_{t}^{B}-X_{t}^{0}-\pi_{t}^{B}\right)}$ is an exponential martingale under the optimal measure $Q^{0}$ obtained from the solution of Merton's problem.

Proof. The same argument used in the proof of Proposition 3.1 shows that

$$
\Lambda_{t}^{0}=\frac{e^{-\gamma\left(X_{t}^{0}-c_{t}^{0}\right)}}{e^{-\gamma\left(x-c_{0}^{0}\right)}}
$$

Therefore 


$$
e^{-\gamma\left(X_{t}^{B}-X_{t}^{0}-\pi_{t}^{B}\right)}=\frac{e^{-\gamma\left(X_{t}^{B}-c_{t}^{B}\right)}}{e^{-\gamma\left(X_{t}^{0}-c_{t}^{0}\right)}}=\Lambda_{t}^{B} / \Lambda_{t}^{0}
$$

It is then clear that $e^{-\gamma\left(X_{t}^{B}-X_{t}^{0}-\pi_{t}^{B}\right)}$ is the density for the measure change from $Q^{0}$ to $Q^{B}$ and therefore an exponential $Q^{0}$-martingale.

Finally, our final proposition for this section generalizes a decomposition obtained in Musiela and Zariphopoulou (2004): the final payoff $B\left(Y_{T}, V_{T}\right)$ can be written as the sum of the indifference price at time 0 , the wealth obtained by trading according to the optimal hedging portfolio and a term corresponding to the unhedgeable risk associated with the process $Y_{t}$.

Proposition 3.4. Suppose the conditions of Proposition 3.1 apply to both $c^{B}$ and $c^{0}$. Then for any $t \in[0, T]$, the payoff $B=B\left(Y_{T}, V_{T}\right)$ admits the decomposition

$$
B=\pi_{t}^{B}+\int_{t}^{T}\left(\frac{b \rho}{\sqrt{Y_{t}}} \partial_{y} \pi_{u}^{B}\right) \frac{\mathrm{d} S_{u}}{S_{u}}+\bar{\rho} \int_{t}^{T} b \partial_{y} \pi_{u}^{B} \mathrm{~d} Z_{u}^{0}-\frac{1}{2} \gamma \bar{\rho}^{2} \int_{t}^{T} b^{2}\left(\partial_{y} \pi_{u}^{B}\right)^{2} \mathrm{~d} u
$$

Here $\mathrm{d} Z_{t}^{0}=\mathrm{d} Z_{t}+v_{t}^{0} \mathrm{~d} t$ defines a Brownian motion under $Q^{0}$, and we adopt the notation $\partial_{y} \pi_{t}^{B}$ for the process $\partial_{y} \pi\left(t, Y_{t}, V_{t}\right)$.

Proof. From (27) we have that

$$
\begin{aligned}
\mathrm{d} \pi_{t}^{B} & =d\left(X_{t}^{B}-X_{t}^{0}\right)-\frac{1}{\gamma}\left[\frac{\left(v_{t}^{B}+v_{t}^{0}\right)\left(v_{t}^{B}-v_{t}^{0}\right)}{2} \mathrm{~d} t+\left(v_{t}^{B}-v_{t}^{o}\right) \mathrm{d} Z_{t}\right] \\
& =d\left(X_{t}^{B}-X_{t}^{0}\right)+b \bar{\rho} \partial_{y} \pi_{t}^{B} \mathrm{~d} Z_{t}^{0}-\frac{\gamma}{2} b^{2} \bar{\rho}^{2}\left(\partial_{y} \pi_{t}^{B}\right)^{2} \mathrm{~d} t .
\end{aligned}
$$

From the functional form for the optimal portfolios $H^{B}$ and $H^{0}$ obtained in Proposition 3.1 we obtain

$$
d\left(X_{t}^{B}-X_{t}^{0}\right)=\left(H_{t}^{B}-H_{t}^{0}\right) \mathrm{d} S_{t}=\frac{b \rho}{S_{t} \sqrt{Y_{t}}} \partial_{y} \pi_{t}^{B} \mathrm{~d} S_{t} .
$$

Substitution into (28) and integration from $t$ to $T$ completes the proof.

\section{Pricing Formulas}

For the special case of bounded claims of the form $B=B\left(Y_{T}\right)$ and constant volatility function $\sigma$ (that is, corresponding to the non-traded asset model mentioned in the previous section), it has been observed by a number of authors (see Henderson and Hobson, 2002; Henderson, 2003; Musiela and Zariphopoulou, 2004; and Monoyios, 2006) that the certainty equivalent partial differential equation (23) can be linearized by a Hopf-Cole transformation. The Feynman-Kac representation of solutions to this new equation can then be used to derive an expression for the certainty equivalent in terms of an expectation with respect to the minimal martingale measure $\tilde{Q}$. In the present section, we provide a duality argument that extends those results to produce more general Feynman-Kac type formulas for claims of the form $B=B\left(Y_{T}\right.$, 
$\left.V_{T}\right)$ in the stochastic volatility setting (1). These formulas are an immediate consequence of the following martingale result:

Theorem 4.1. Consider a claim of the form $B=B\left(Y_{T}, V_{T}\right)$ and suppose that $c^{B}$ satisfies the conditions of Proposition 3.1. Then the process

$$
\Xi_{t}=e^{\gamma \bar{\rho}^{2} c_{t}^{B}} e^{-\frac{1}{2} \int_{0}^{t} \bar{\rho}^{2} \lambda_{s}^{2} \mathrm{~d} s}
$$

is a $\tilde{Q}$ - martingale.

Proof. It follows from Itô's formula that

$$
\mathrm{d} c_{t}^{B}=\left(\partial_{t} c^{B}+a \partial_{y} c^{B}+\frac{1}{2} b^{2} \partial_{y y}^{2} c^{B}+f \partial_{v} c^{B}\right) \mathrm{d} t+b \partial_{y} c^{B}\left(\rho \mathrm{d} W_{t}+\bar{\rho} \mathrm{d} Z_{t}\right),
$$

which in turn implies that

$$
\begin{aligned}
\frac{\mathrm{d} \Xi_{t}}{\Xi_{t}}= & \gamma \bar{\rho}^{2} \mathrm{~d} c_{t}^{B}+\frac{1}{2} \gamma^{2} \bar{\rho}^{4}\left(\mathrm{~d} c_{t}^{B}\right)^{2}-\frac{1}{2} \bar{\rho}^{2} \lambda_{t}^{2} \mathrm{~d} t \\
= & \gamma \bar{\rho}^{2}\left[\left(\partial_{t} c^{B}+a \partial_{y} c^{B}+\frac{1}{2} b^{2} \partial_{y y}^{2} c^{B}+f \partial_{v} c^{B}-\frac{\lambda_{t}^{2}}{2 \gamma}\right.\right. \\
& \left.\left.+\frac{1}{2} \gamma \bar{\rho}^{2} b^{2}\left(\partial_{y} c^{B}\right)^{2}\right) \mathrm{~d} t+\partial_{y} c^{B} b\left(\rho \mathrm{d} W_{t}+\bar{\rho} \mathrm{d} Z_{t}\right)\right] \\
= & \gamma \bar{\rho}^{2}\left[\left(\partial_{t} c^{B}+\left(a-b \rho \lambda_{t}\right) \partial_{y} c^{B}+\frac{1}{2} b^{2} \partial_{y y}^{2} c^{B}+f \partial_{v} c^{B}-\frac{\lambda_{t}^{2}}{2 \gamma}\right.\right. \\
& \left.\left.+\frac{1}{2} \gamma \bar{\rho}^{2} b^{2}\left(\partial_{y} c^{B}\right)^{2}\right) \mathrm{~d} t+\partial_{y} c^{B} b\left(\rho \mathrm{d} \tilde{W}_{t}+\bar{\rho} \mathrm{d} Z_{t}\right)\right],
\end{aligned}
$$

where we have introduced the $\tilde{Q}$ Brownian motion $\mathrm{d} \tilde{W}_{t}=\mathrm{d} W_{t}+\lambda_{t} \mathrm{~d} t$. If we now recall equation (23) and the fact that $\Lambda_{t}^{B}$ is a martingale under the physical measure $P$, we obtain that the drift term above vanishes, so that $\Xi$ is an exponential local martingale under the measure $\tilde{Q}$. To show that $E^{\tilde{Q}}\left[\Xi_{T}\right]=1$ and thus $\Xi$ is a true $\tilde{Q}$-martingale, we first observe that the processes $\lambda_{t}, v_{t}^{B}$ are $\mathcal{F}_{t}^{M^{1}}$-adapted, where

$$
M_{t}^{1}=\rho W_{t}+\bar{\rho} Z_{t}, M_{t}^{2}=-\bar{\rho} W_{t}+\rho Z_{t}
$$

are independent $P$-Brownian motions. This then allows us to use the following simple result: then

Lemma 4.2. If $\ell_{t}$ is a $\mathcal{F}_{t}^{M^{1}}$-adapted process such that and $P\left[\int_{0}^{T} \ell_{t}^{2} \mathrm{~d} t<\infty\right]=1$,

$$
E\left[L_{T} \mid \mathcal{F}_{T}^{M^{1}}\right]=1, \quad \text { Pa.s. }
$$

where $L_{t}$ is the stochastic exponential satisfying $\mathrm{d} L_{t}=\ell_{t} L_{t} \mathrm{~d} M_{t}^{2}$ with $L_{0}=1$. 
If we now define $\tilde{\Lambda}_{t}:=E\left[\frac{\mathrm{d} \tilde{Q}}{\mathrm{~d} P} \mid \mathcal{F}_{t}\right]$, then the two stochastic exponentials $\tilde{\Lambda}_{t} \Xi_{t}$ and $\Lambda_{t}^{B}$ can be thought of as Radon-Nikodym derivatives of measure changes away from the measure $P$. Direct computation using (17), (18) and (19) then verifies the following factorizations:

$$
\tilde{\Lambda}_{t} \Xi_{t}=\Lambda_{t}^{1} \Lambda_{t}^{2}, \quad \Lambda_{t}^{B}=\Lambda_{t}^{1} \Lambda_{t}^{3}
$$

where

$$
\begin{aligned}
& \mathrm{d} \Lambda_{t}^{1}=-\Lambda_{t}^{1}\left(\bar{\rho} v_{t}^{B}+\rho \lambda_{t}\right) \mathrm{d} M_{t}^{1}, \\
& \mathrm{~d} \Lambda_{t}^{2}=\Lambda_{t}^{2} \bar{\rho} \lambda_{t} \mathrm{~d} M_{t}^{2}, \\
& \mathrm{~d} \Lambda_{t}^{3}=\Lambda_{t}^{3}\left(\bar{\rho} \lambda_{t}-\rho v_{t}^{B}\right) \mathrm{d} M_{t}^{2} .
\end{aligned}
$$

By applying Lemma 4.2 to the $\mathcal{F}_{t}^{W^{1}}$-adapted processes $\bar{\rho} \lambda_{t}$ and $\bar{\rho} \lambda_{t}-\rho v_{t}^{B}$ one finds

$$
\begin{aligned}
E^{\tilde{Q}}\left[\Xi_{T}\right] & =E\left[\Lambda_{T}^{1} \Lambda_{T}^{2}\right]=E\left[\Lambda_{T}^{1} E\left[\Lambda_{T}^{2} \mid \mathcal{F}_{T}^{W^{1}}\right]\right] \\
& =E\left[\Lambda_{T}^{1}\right]=E\left[\Lambda_{T}^{1} E\left[\Lambda_{T}^{3} \mid \mathcal{F}_{T}^{W^{1}}\right]\right]=E\left[\Lambda_{T}^{B}\right]=1 .
\end{aligned}
$$

The required conditions $P\left[\int_{0}^{T} \bar{\rho}^{2} \lambda_{t}^{2} \mathrm{~d} t<\infty\right]=1$ and $P\left[\int_{0}^{T}\left(\bar{\rho} \lambda_{t}-\rho v_{t}^{B}\right)^{2} \mathrm{~d} t<\infty\right]=1$ are consequences of Theorem 3.1 of Wong and Heyde (2004) and the fact that $\Lambda_{t}^{B}$ is a martingale.

By the martingale condition on $\Xi_{t}$, it is immediate to verify that

$$
e^{\gamma \bar{\rho}^{2} c_{t}^{B}}=E_{t}^{\tilde{Q}}\left[e^{\gamma \bar{\rho}^{2} B\left(Y_{T}, V_{T}\right)} e^{-\frac{1}{2} \int_{t}^{T} \bar{\rho}^{2} \lambda_{s}^{2} \mathrm{~d} s}\right] .
$$

Therefore

$$
c_{t}^{B}=\frac{1}{\gamma \bar{\rho}^{2}} \log E_{t}^{\tilde{Q}}\left[e^{\gamma \bar{\rho}^{2} B\left(Y_{T}, V_{T}\right)} e^{-\frac{1}{2} \int_{t}^{T} \bar{\rho}^{2} \lambda_{s}^{2} \mathrm{~d} s}\right]
$$

and, by the same argument

$$
c_{t}^{0}=\frac{1}{\gamma \bar{\rho}^{2}} \log E_{t}^{\tilde{Q}}\left[e^{-\frac{1}{2} \int_{t}^{T} \bar{\rho}^{2} \lambda_{s}^{2} \mathrm{~d} s}\right] .
$$

This then proves the most important consequence of Theorem 4.1, namely, a Feynman-Kac type representation for the indifference price of volatility claims. A similar formula has been derived independently by Monoyios (2006).

Corollary 4.3. Consider a claim of the form $B=B\left(Y_{T}, V_{T}\right)$ and suppose that both $c^{B}$ and $c^{0}$ satisfy the conditions of Proposition 3.1. Then, the indifference price for a volatility claim $B=B\left(Y_{T}, V_{T}\right)$ at time $t<T$ can be written as

$$
\pi_{t}^{B}=\frac{1}{\gamma \bar{\rho}^{2}} \log \left(\frac{E_{t}^{\tilde{Q}}\left[e^{\gamma \bar{\rho}^{2} B\left(Y_{T}, V_{T}\right)} e^{-\frac{1}{2} \int_{t}^{T} \bar{\rho}^{2} \lambda_{s}^{2} \mathrm{~d} s}\right]}{E_{t}^{\tilde{Q}}\left[e^{-\frac{1}{2} \int_{t}^{T} \bar{\rho}^{2} \lambda_{s}^{2} \mathrm{~d} s}\right]}\right)
$$




\section{The Variance Swap}

As an explicit application of the previous section, we consider the indifference pricing for $q$ units of the variance swap, defined by the total discounted payoff

$$
B_{T}=q e^{-r T} \int_{0}^{T} \sigma_{t}^{2} \mathrm{~d} t
$$

Various papers, notably Carr et al. (2005) and Friz and Gatheral (2005), have studied the pricing of these increasingly traded derivatives by a robust replication argument. Their method relies on exactly hedging the volatility derivative by taking positions in an infinity of equity options, and is therefore suited to mature markets, where calls and puts on the underlying asset are extensively traded. In contrast to their method for an "over-complete" type of market, our method provides a rational hedge for volatility derivatives that involves trading only the underlying asset itself, and is therefore better suited for new and emerging markets.

The next subsections introduce two classes of stochastic volatility models based on an auxiliary Cox-Ingersoll-Ross process $\Psi_{t}$ governed by the SDE

$$
\mathrm{d} \Psi_{t}=\left(A-B \Psi_{t}\right) \mathrm{d} t+C \sqrt{\Psi_{t}} \mathrm{~d} M_{t}, \quad \Psi_{0}=\psi_{0},
$$

where $M_{t}$ is a $P$-Brownian motion and the constant parameters $(A, B, C)$ satisfy $2 A \geqslant C^{2}$ which guarantees that 0 is a non-attainable boundary, and $B \geqslant 0$ (note that $B>0$ guarantees that the process mean-reverts).

As we shall see, the right sides of formulas (34), (32), (33), which we denote by $\hat{\pi}_{t}^{B}, \hat{c}_{t}^{B}, \hat{c}_{t}^{0}$, are candidates for the indifference price and certainty equivalents of the variance swap, and are explicit inboth models in terms of the following Laplace transform:

$$
G\left(T, \psi_{0} ; d_{1}, d_{2}\right)=E\left[e^{-d_{1} \int_{0}^{T} \Psi_{s} \mathrm{~d} s-d_{2} \int_{0}^{T} \Psi_{s}^{-1} \mathrm{~d} s}\right]
$$

This function has been studied in Hurd and Kuznetsov (2006), where the following explicit formula in terms of the confluent hypergeometric function ${ }_{1} F_{1}$ was presented:

Proposition 5.1. Suppose $d_{1} \geq-\frac{B^{2}}{2 C^{2}}$ and $d_{2} \geq-\frac{\alpha^{2} C^{2}}{8}$, where $\alpha=2 A / C^{2}-1$. Then

$$
\begin{aligned}
G\left(T, x ; d_{1}, d_{2}\right) & =\exp \left(-x\left(v_{1}+\frac{\gamma_{T}\left(-v_{1}\right)}{\gamma_{T}-v_{1}} e^{-\left(b+2 v_{1} C^{2}\right) T}\right)\right) \\
& \times e^{-\left(A v_{1}+B v_{2}+C^{2} v_{1} v_{2}\right) T} x^{v_{2}}\left(\gamma_{T}-v_{1}\right)^{-\alpha-v_{2}-1} \gamma_{T}^{\alpha+2 v_{2}+1} \\
& \times \frac{\Gamma\left(\alpha+v_{2}+1\right)}{\Gamma\left(\alpha+2 v_{2}+1\right)} 1 F_{1}\left(v_{2}, \alpha+2 v_{2}+1 ;-\frac{\gamma_{T}^{2} x e^{-\left(B+2 v_{1} C^{2}\right) T}}{\gamma_{T}-v_{1}}\right) .
\end{aligned}
$$

where

$$
v_{1}=\frac{1}{2}\left(-\beta+\sqrt{\beta^{2}+\frac{8 d_{1}}{C^{2}}}\right), v_{2}=\frac{1}{2}\left(-\alpha+\sqrt{\alpha^{2}+\frac{8 d_{2}}{C^{2}}}\right)
$$


and $\gamma_{T}=\left(\beta+2 v_{1}\right)\left(1-e^{-\left(B+2 v_{1} C^{2}\right) T}\right)^{-1}$.

It remains to be shown that these candidate functions, which are smooth and solve (23), are indeed equal to the true optimizers $\pi_{t}^{B}, c_{t}^{B}, c_{t}^{0}$. However, known verification results for controlled Markov process, such as those in Fleming and Soner (1993), do not apply here, since they rely on stronger boundedness conditions.

\section{Heston Model}

The Heston model (Heston, 1993) has the form (1) where $\sigma_{t}^{2}=Y_{t}$ has CIR dynamics given by (36) for the Brownian motion $M_{t}=\rho W_{t}+\bar{\rho} Z_{t}$ and coefficient functions

$$
a\left(t, Y_{t}\right)=A-B Y_{t}, \quad b\left(t, Y_{t}\right)=C \sqrt{Y_{t}} .
$$

We now proceed to verify that the standing assumptions of Section 1 are satisfied by the variance swap in this model. First observe that when $B_{T}$ is given by (35), we have

$$
E\left[e^{\alpha B_{T}}\right]=G\left(T, Y_{0} ;-\alpha q e^{-r T}, 0\right)
$$

Using Proposition 5.1, we see that Assumption 1.2 is verified for $e^{-r T} \gamma q<\frac{B^{2}}{2 C^{2}}$. This also provides a condition on $\int_{0}^{T} \sqrt{Y_{S}} \mathrm{~d} W_{s}$ that guarantees Assumption 1.1. Finally, following Section 6 of Cheridito, Filipović and Yor (2005), it can be shown that $E[\mathrm{~d} \tilde{Q} / \mathrm{d} P]=1$, that the finite relative entropy condition holds, and that $Y_{t}$ has CIR dynamics with parameters $\tilde{A}=A-\rho C(\mu-r), B, C$ under $\tilde{Q}$, all provided the boundary non-attainment condition $2 \tilde{A} \geq C^{2}$ holds under $\tilde{Q}$.

One can now prove the following result by substitution of the formulas for $B_{T}$ and $\lambda_{t}^{2}$ into (34), (32), (33) and then verifying the conditions of Proposition 5.1 one more time.

Proposition 5.2. Assumptions 1.1, 1.2, 1.3 hold if $e^{-r T} \gamma q<\frac{B^{2}}{2 C^{2}}$ and $A-\rho C(\mu-r) \geqslant C^{2} / 2$, in which case $Y_{t}$ has CIR dynamics under $\tilde{Q}$ with parameters $A-\rho C(\mu-r), B, C$. Moreover, the time $t=0$ candidate seller's indifference price for $q$ units of the variance swap in the Heston model is then finite and given by

$$
\hat{\pi}^{B}(0, y)=\frac{1}{\gamma \bar{\rho}^{2}} \log \left(\frac{G\left(T, y ; d_{1}, d_{2}\right)}{G\left(T, y ; 0, d_{2}\right)}\right)
$$

where $d_{1}=-q \gamma \bar{\rho}^{2} e^{-r T}, d_{2}=\frac{1}{2} \bar{\rho}^{2}(\mu-r)^{2}$.

\section{Reciprocal Heston Model}

We now exploit the $\Psi \rightarrow \Psi^{-1}$ symmetry inherent in (37) and define a new model of the form (1) with

$$
\sigma_{t}^{2}=Y_{t}=\frac{1}{\Psi_{t}},
$$


where $\Psi_{t}$ is a CIR process of the form (36) driven by the Brownian motion $M_{t}^{1}$. This model, which we call "reciprocal Heston", has a number of desirable properties. The reciprocal of a CIR process, being positive and mean reverting, is areasonable model for stochastic squared volatility that has recently been studied by Chacko and Viciera (2005). The reciprocal CIR process has also been used by Ahn and Gao (1999) as a term structure model for which bond prices can be computed analytically.

As before, we use Proposition 5.1 to find conditions on the model that guarantee the assumptions of Section 1 hold. Since for the variance swap (35) in this model we now have

$$
E\left[e^{\alpha B_{T}}\right]=G\left(T, \Psi_{0} ; 0,-\alpha q e^{-r T}\right),
$$

we see that Assumption 1.2 is verified for $\gamma q e^{-r T}<\frac{C^{2}}{2}\left(\frac{A}{C^{2}}-\frac{1}{2}\right)^{2}$. Once more, this in turn provide a condition on $\int_{0}^{T} \sqrt{Y_{s}} \mathrm{~d} W_{s}$ that guarantees Assumption 1.1. Finally, as in the previous section, we can prove $E[\mathrm{~d} \tilde{Q} / \mathrm{d} P]=1$, the finite relative entropy condition, and that $Y_{t}$ has CIR dynamics with parameters $A, \tilde{B}=B-\rho C(\mu-r), C$ under $\tilde{Q}$, all provided the condition $\tilde{B} \geq 0$ holds under $\tilde{Q}$.

Similarly to Proposition 5.2, we obtain the following result for a variance swap in the reciprocal Heston model:

Proposition 5.3. Assumptions 1.1, 1.2, 1.3 hold if $e^{-r T} \gamma \underset{\sim}{q}<\frac{C^{2}}{2}\left(\frac{A}{C^{2}}-\frac{1}{2}\right)^{2}$ and $B \geqslant \rho C(\mu-r)$, in which case $Y_{t}$ has CIR dynamics under $\tilde{Q}$ with parameters $A, \tilde{B}=B-\rho C(\mu-r), C$. Moreover, the time $t=0$ candidate seller's indifference price for $q$ units of the variance swap in the reciprocal Heston model is finite and given by

$$
\hat{\pi}^{B}(0, y)=\frac{1}{\gamma \bar{\rho}^{2}} \log \left(\frac{G\left(T, \Psi_{0} ; d_{1}, d_{2}\right)}{G\left(T, \Psi_{0} ; d_{1}, 0\right)}\right)
$$

where $d_{1}=\frac{1}{2} \bar{\rho}^{2}(\mu-r)^{2}, d_{2}=-q \gamma \bar{\rho}^{2} e^{-r T}$.

\section{Numerics of Variance Swap}

While it is not our purpose here to explore the details of the above formulas, it is nonetheless worthwhile to consider briefly how indifference prices compare to other pricing methods for the variance swap. We take the Bakshi et al. (1997) parameters for the Heston model: $\mu=0.127, \rho=-0.28, r=0.04, A=0.04, B=1.16, C=0.2, Y_{0}=A$ / $B{ }^{1}$ Without loss of generality, we may take $\gamma=1, x=0$. Figure 1 shows the indifference price for $q=1$ units of the variance swap, as a function of time to maturity $T$, for several values of $\rho$, illustrating that as $|\rho| \rightarrow 1$, the price converges to the complete market value computed when $|\rho|=1$. Figure 2 shows the per unit indifference price of a $T=1$ variance swap as a function of $q$, again for various values of $\rho$. This graph illustrates that the per unit price is increasing and convex in $q$. In fact, all curves eventually diverge as $q$ increases. Computations were done in Mathematica. Algebraic differentiation within Mathematica permits the evaluation of the hedging strategy function $h$, as well as any greeks of interest. 
unit price vs $T$, various rho values

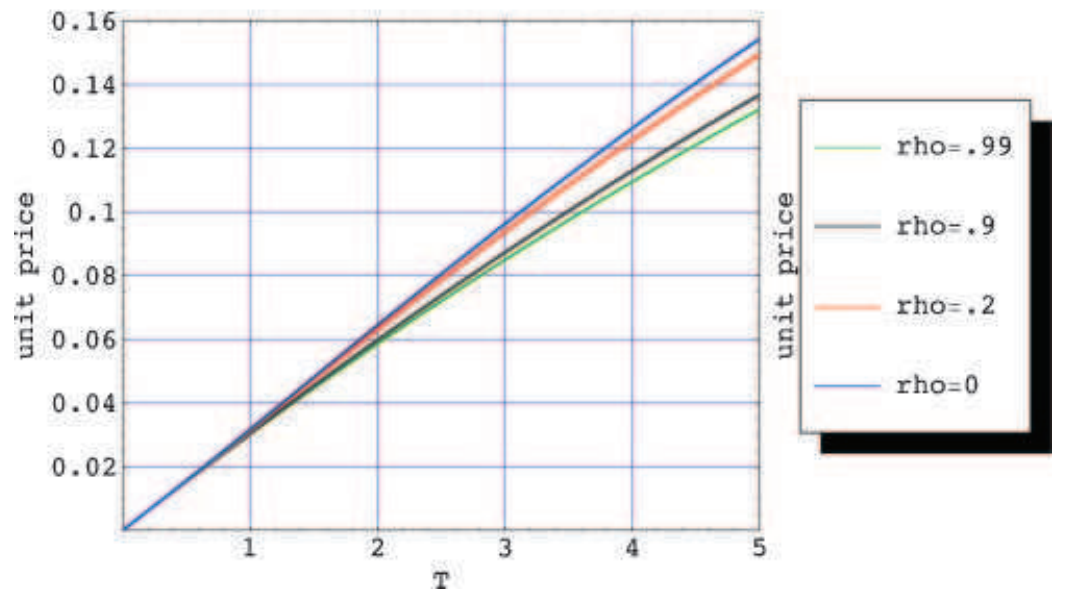

Figure 1. Indifference price of $q=1$ units of the variance swap as a function of time to maturity $T$, for different values of $\rho$.

\section{unit price vs $\mathrm{q}$, various rho values}

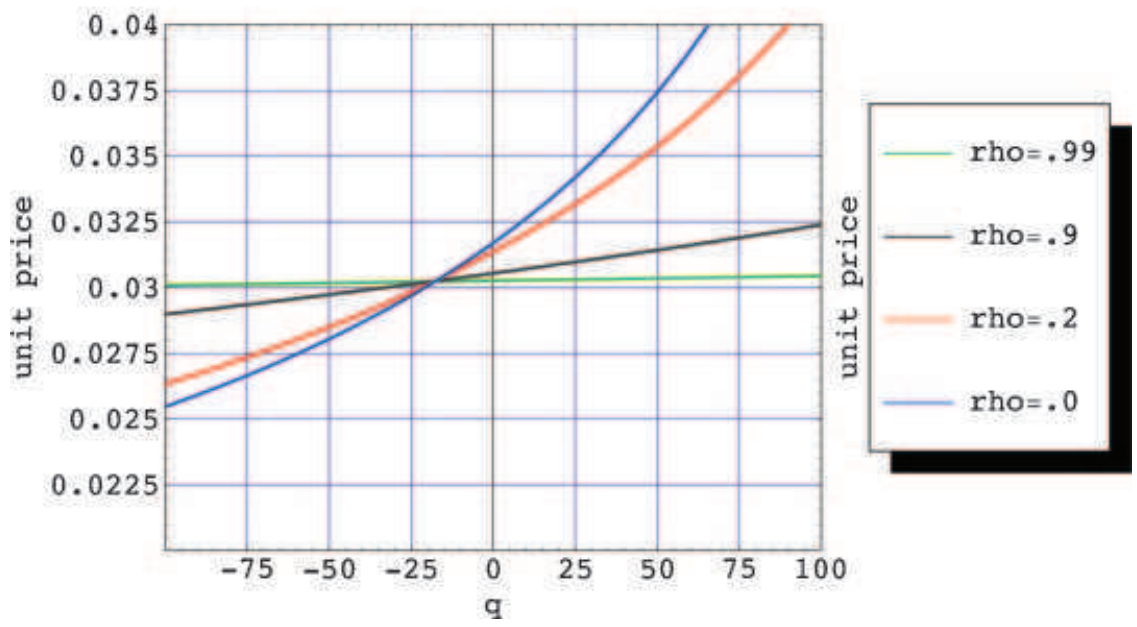

Figure 2. Indifference price per unit for the $T=1$ variance swap as a function of $q$, for different values of $\rho$.

\section{Conclusions}

This paper has developed the theory of indifference pricing and hedging for volatility derivatives in a class of stochastic volatility models general enough to include industry relevant examples. The main theoretical result, Theorem 4.1, yields a very useful nonlinear Feynman-Kac representation for indifference prices with the character of formulas found by Musiela and Zariphopoulou (2004) and others. We 
then applied this result to find closed formulas for candidate indifference prices of variance swaps in the Heston model and in a new model we call the reciprocal Heston model. While a verification result tailored for these examples is not known to us, and is a topic for future research, our candidate functions are smooth classical solutions of the associated HJB equation and are easy to compute, therefore opening the possibility for detailed numerical exploration of these and related financially important indifference prices.

\section{Note}

${ }^{1}$ We reduce the "volatility of volatility" parameter $C$ by a factor of 2 to ensure the no-hitting condition $2 A \geqslant C^{2}$.

\section{References}

Ahn, B.-H. and Gao, B. (1999) A parametric nonlinear model of term structure dynamics, Revue of Financial Studies, 12, pp. 721-762.

Bakshi, G. et al. (1997) Empirical performance of alternative option pricing models, Journal of Finance, 52 , pp. 2003-2049.

Becherer, D. (2001) Rational Hedging and Valuation with Utility-based Preferences. PhD thesis, Technischen Universitat Berlin.

Becherer, D. (2004) Utility-indifference hedging and valuation via reaction-diffusion systems, Proceedings of Royal Society, 460(2041), pp. 27-51.

Carr, P. et al. (2005) Pricing options on realized variance, Finance Stochastics, pp. 453-475, 2005.

Chacko, G. and Viciera, L. (2005) Dynamic consumption and portfolio choice with stochastic volatility, Revue of Financial Studies, 18, pp. 1369-1402.

Cheridito, P. et al. (2005) Equivalent and absolutely continuous measure changes for jump-diffusion processes, Annals of Applied Probability, 15, pp. 1713-1732.

Cvitanić, J. et al. (2001) Utility maximization in incomplete markets with random endowment, Finance Stochastics, 5(2), pp. 259-272.

Delbaen, F. et al. (2002) Exponential hedging and entropic penalties, Mathematical Finance, 12(2), pp. 99-123.

Friz, P. and Gatheral, J. (2005) Pricing volatility derivatives as inverse problem, Quantitative Finance, 5.

Henderson, V. (2002) Valuation of claims on nontraded assets using utility maximization, Mathematical Finance, 12(4), pp. 351-373.

Heston, S. L. (1993) A closed-form solution for options with stochastic volatility with applications to bond and currency options, Revue of Financial Studies, 6(2), pp. 327-343.

Hodges, S. D. and Neuberger, A. (1989) Optimal replication of contingent claims under transaction costs, Revue of Futures Markets, 8, pp. 222-239.

Hurd, T. R. and Kuznetsov, A. (2006) Explicit formulas for laplace transforms of stochastic integrals. Working paper, February. Available at: http://www.math.mcmaster.ca/tom/StochIntHurdKuzn.pdf

Kabanov, Y. M. and Stricker, C. (2002) On the optimal portfolio for the exponential utility maximization: remarks to the six-author paper, Mathematical Finance, 12(2), pp. 125-134.

Monoyios, M. (2006) Characterisation of optimal dual measures via distortion, To appear in Decisions in Economics and Finance.

Musiela, M. and Zariphopoulou, T. (2004) An example of indifference prices under exponential preferences, Finance Stochastics, 8(2), pp. 229-239.

Owen, M. P. (2002) Utility based optimal hedging in incomplete markets, Annals of Applied Probability, 12(2), pp. 691-709.

Sircar, R. and Zariphopoulou, T. (2005) Bounds and asymptotic approximations for utility prices when volatility is random, SIAM Journal of Control and Optimization, 43, pp. 1328-1353.

Wong, B. and Heyde, C. C. (2004) On the martingale property of stochastic exponentials, Journal of Applied Probability, 41, pp. 654-664. 